\title{
Mapas de competencias genéricas del bachillerato
}

\author{
High-School Generic Competence Maps
}

\author{
Rafael Morales Gamboa \\ Universidad de Guadalajara. Guadalajara, México \\ rmorales@suv.udg.mx \\ María Inez González Navarro \\ Universidad de Guadalajara. Guadalajara, México \\ inez.gonzalez@suv.udg.mx \\ Yessenia Reyes Sánchez \\ Universidad de Guadalajara. Guadalajara, México \\ yesenia.reyes@alumno.udg.mx
}

\section{Resumen}

Se presentan los resultados del análisis de las competencias genéricas establecidas para el Sistema Nacional de Bachillerato de México y la codificación de los resultados en la forma de mapas de competencias usando un lenguaje susceptible de ser procesado automáticamente por computadoras. Específicamente, se presentan una revisión de literatura sobre la representación computacional de competencias, un mapa de competencias genéricas con relaciones de subsume/esun e incluye/parte-de y la aplicación del mapa para identificar la presencia de competencias genéricas en el diseño de cursos de bachillerato en el Sistema de Universidad Virtual de la Universidad de Guadalajara.

Palabras clave. Competencia, mapa de competencias, representación computacional, competencia genérica, etiquetado por competencias.

\begin{abstract}
We present the results of an analysis of the generic competences established for the National High School System of Mexico, and the coding of the results in the form of competence maps using a language that can be automatically processed by computers. Specifically, we present a literature review on the computational representation of competencies, a map of generic competencies with subsume/is-a and includes/part-of relationships, and the application of the competences map to identify the presence of generic competences in the design of high school courses in the Virtual University System of the University of Guadalajara.
\end{abstract}

Keywords. Competence, competences map, computational representation, tracing of competence development, generic competence. 


\section{Introducción}

La educación en línea ha traído muchos cambios en los procesos educativos de la educación formal, comenzando con el reemplazo del aula, como espacio físico cerrado en donde se converge de manera síncrona, por un entorno digital en donde se opera generalmente de manera asíncrona ${ }^{1}$. Se ha generado una división entre quienes diseñan los cursos y quienes los imparten, pues si bien en la educación en presencia física el libro de texto determina en buena medida los contenidos y la forma de la enseñanza, el docente tiene más libertad para realizar las modificaciones que considere pertinentes. La población objetivo "tradicional" de la educación a distancia, adultos con necesidad de regularizar su formación profesional, ya sea por los conocimientos o por el certificado, ha facilitado la transición hacia modelos educativos orientados hacia el desarrollo de competencias, de modo que los programas, los cursos y las actividades se diseñan, al menos en principio, para promover en los alumnos el desarrollo de competencias (Moreno Castañeda \& Pérez Alcalá, 2010).

Por otro lado, hay varios aspectos de la educación formal que históricamente se han mantenido inamovibles en la transición hacia la educación en línea, como es el diseño educativo organizado en programas, cursos, unidades y actividades, así como la segmentación de la población estudiantil en cursos y grupos ${ }^{2}$. Sin embargo, para propósitos de este artículo, se hace énfasis solamente en uno: el certificado donde generalmente se registran los cursos que el estudiante ha tomado en un programa de estudios y las calificaciones que ha obtenido en cada uno de ellos.

El certificado de estudios ofrece una imagen general del desempeño del estudiante a lo largo de sus estudios, así como algunas pistas sobre su vocación, reflejada en calificaciones diferencias por áreas de conocimiento y la selección de cursos optativos con los que ha complementado su formación. Sin embargo, la mayoría de los cursos tienen títulos tan generales, como Ética y Política o Matemática avanzada, que nos dicen muy poco sobre las competencias desarrolladas por los estudiantes en cada uno de ellos. Por otra parte, es poco creíble que todas y cada una de las competencias atendidas en un curso se hayan desarrollado al mismo nivel, de modo que la calificación obtenida en el curso por el estudiante nos habla, cuando mucho, de una tendencia central.

Inferir el grado de desarrollo de las competencias genéricas a partir del contenido del certificado de estudios es todavía más difícil, pues no suelen estar asociadas a un curso particular ni reflejadas en su título; de modo que, para darnos una idea de su desarrollo, lo mejor que tenemos es el promedio general y la suposición de que todas ellas fueron atendidas suficientemente en todos y cada uno de los cursos registrados en el

1 Si bien la condición actual de pandemia y su correspondiente contingencia sanitaria han cambiado la proporción entre asincronía y sincronía.

2 Al menos en México, 'la educación a distancia [en línea] se relaciona más con las estrategias metodológicas y tecnológicas que posibilitan la entrega de contenidos educativos, y con la comunicación entre los participantes de un proceso educativo determinado que no coinciden en tiempo y lugar' (Moreno Castañeda, 2015).

Mapas de competencias genéricas del bachillerato. Rafael Morales, Morales Gamboa, González Navarro y Reyes Sánchez.

Página 2 de 27 


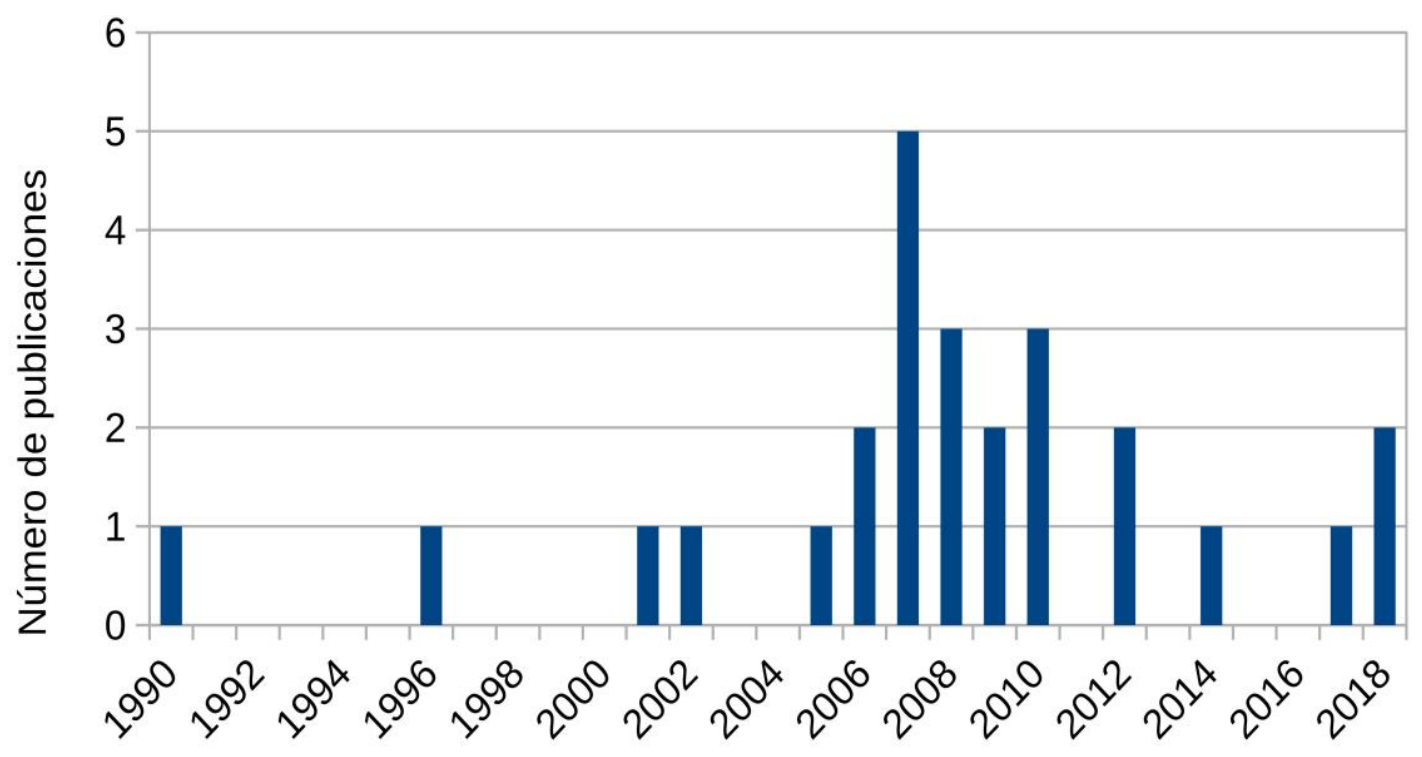

Año

certificado. De modo que, si realmente se está interesado en conocer con mayor detalle el desarrollo de las competencias genéricas de un estudiante, será necesario revisar el plan de estudios y el diseño de los cursos, preguntarle a todos y cada uno de sus maestros o, en el caso de la educación en línea, desenterrar el respaldo de los cursos y revisar las actividades del estudiante en los mismos.

Este problema tiene solución en la educación en línea si se considera el hecho de que un sistema gestor del aprendizaje suele contener la información de lo ocurrido en un curso, tanto del comportamiento del estudiante como de la mediación y evaluación de parte del docente, lo cual puede ser traducido a información sobre el desarrollo de competencias por los estudiantes. Para ello es indispensable proveer al sistema computacional de información detallada de las competencias que conforman la base del diseño de los cursos, o representaciones de competencias, de su relación con las distintas actividades en los mismos y de la manera en que el desempeño del estudiante, generalmente observado desde la perspectiva del docente, puede ser interpretado como evidencia del estado de sus competencias haciendo uso de técnicas de Inteligencia Artificial.

En el Sistema de Universidad Virtual de la Universidad de Guadalajara se ha estado desarrollando un proyecto precisamente en esa línea (Medina Flores \& Morales Gamboa, 2017; Morales Gamboa \& Sucar, 2019; Rodríguez Yépez \& Morales Gamboa, 2019) el cual necesita ser alimentado con representaciones computacionales de las competencias a ser desarrolladas en los distintos programas educativos, así como con las relaciones entre estas competencias y las distintas actividades que realiza el estudiante en sus cursos. Decidimos comenzar por las competencias genéricas del

Mapas de competencias genéricas del bachillerato. Rafael Morales, Morales Gamboa, González Navarro y Reyes Sánchez.

Página 3 de 27 
programa de Bachillerato General por Áreas Interdisciplinarias dado que estas son compartidas no solamente con el programa de bachillerato presencial ofrecido por la Universidad de Guadalajara en General sino con todos los programas de bachillerato adscritos al Sistema Nacional de Bachillerato de México.

En la siguiente sección se hace una revisión del estado del arte en representación computacional de competencias. Posteriormente, se presenta el esquema de representación seleccionado para este proyecto, cómo se ha aplicado a la representación computacional de las competencias genéricas del Sistema Nacional de Bachillerato (Secretaría de Educación Pública, 2008, p. 444) y cuáles han sido los resultados. Finalmente, se presentan los resultados obtenidos de observar la presencia de las competencias genéricas en el diseño de una colección de cursos de bachillerato El artículo cierra con conclusiones y propuestas de trabajo a futuro.

\section{Estado del arte}

Se realizó una búsqueda en las bases de datos de referencias Web of Knowledge (webofknowledge.com) y Google Scholar (scholar.google.com) usando términos tales como 'representación de competencias' y 'competencia AND representación computacional', y sus equivalentes en inglés. En la lista de referencias de los documentos relevantes encontrados en esas bases se identificaron otros documentos relevantes y, en algunos casos, se estableció contacto con autores a fin de identificar desarrollos más recientes de su investigación en ese tema. En total, se seleccionaron veintiséis documentos relacionados directamente con el tema, con fechas de publicación entre 1990 y 2018. La muestra la distribución de publicaciones identificas en ese periodo y sugiere que el mayor interés en el tema se dio entre 2006 y 2011, con dieciséis artículos (62\%) identificados en ese periodo.

\section{Propósito}

El interés por la representación de competencias surge de propósitos diversos que se pueden clasificar en cuatro categorías: interoperabilidad, gestión de personal, educación y representación.

En la categoría de interoperabilidad se ubican los trabajos con interés particular en la representación de competencias para el intercambio de información entre sistemas diferentes - plataformas educativas, portafolios, de gestión de recursos humanos, entre otros. Todos ellos giran en torno a la recomendación de IMS Global (IMS Global Learning Consortium, 2002) y el estándar de IEEE (Competency Data Working Group, 2008) para el intercambio de información sobre competencias, a las cuales critican y extienden de maneras diversas (El Asame \& Wakrim, 2018; Karampiperis et al., 2006; D. Sampson et al., 2007; D. Sampson \& Fytros, 2008; D. G. Sampson, 2009; Sitthisak et al., 2007).

En la categoría de gestión de personal se ubican los trabajos enfocados en la gestión de

Mapas de competencias genéricas del bachillerato. Rafael Morales, Morales Gamboa, González Navarro y Reyes Sánchez.

Página 4 de 27 
competencias en el ámbito organizacional (Vasconcelos et al., 2001), ya sea con fines de definición de puestos e identificación de falta de capacitación (Yu \& Zhang, 1990), su relación con la documentación empresarial (Lefebvre et al., 2005), contratación de personal (Lee \& Benati, 2009; Miranda et al., 2017) y el modelado de competencias individuales, grupales y organizacionales (Pépiot et al., 2007).

La categoría de educación engloba trabajos asociados con sistemas tutores inteligentes (Nkambou et al., 1996), con la construcción de infraestructura (Paquette, 2007; Paquette et al., 2006) para la implementación de Learning Design (IMS Global Learning Consortium, 2003), gestión de contenidos para el desarrollo de competencias (Różewski \& Małachowski, 2009), como soporte de un entorno de aprendizaje (Różewski \& Małachowski, 2010) y soporte para el individuo (Biletska et al., 2010) y las instituciones (Cicortas et al., 2008) en el aprendizaje para toda la vida. En esta categoría, el artículo de Stoof et al. (2007) se enfoca a la construcción de mapas de competencias que sirvan para la especificación de perfiles de egreso.

Finalmente, los trabajos en la categoría de representación son aquellas cuyo objetivo se acota a la representación de competencias en sí, sin una aplicación aparente en el texto (García-Barriocanal et al., 2012; May \& Ossendorf, 2014; Ramírez \& Sánchez, 2012; Wang \& Wang, 2018).

\section{Noción de competencia}

En 1973, McClellan arremetió contra las pruebas de inteligencia y actitude, así como las calificaciones en los programas educativos, como predictores de la capacidad de las personas para desempeñarse exitosamente en su trabajo, e introduce el término 'competencia' (competence) como 'un símbolo de una aproximación alternativa a la evaluación de inteligencia' (McClelland, 1973, p. 7) y a los exámenes en los cuales los estudiantes producen respuestas en situaciones controladas en vez de operar en situaciones más reales; posteriormente, hace una distinción entre las competencias propias de ámbitos laborales (o académicos) específicos y aquellas que son útiles en ámbitos más amplios de la vida. Al menos desde entonces, la noción de competencia ha reforzado su asociación directa con la capacidad de hacer, actuar u operar de ciertas maneras en determinadas condiciones, así como con los atributos personales (grupales u organizacionales) que pudieran explicar esa capacidad en individuos, grupos $\mathrm{u}$ organizaciones.

En lo referente a la representación computacional de competencias, en las primeras publicaciones se parte de la noción de competencia como un conjunto de elementos (Nkambou et al., 1996; Yu \& Zhang, 1990), que se expande posteriormente con la inclusión de elementos de desempeño (D. Sampson \& Fytros, 2008) y recientemente se ha generalizado a conjuntos difusos, con grados de pertenencia (Wang \& Wang, 2018). Si bien desde la perspectiva educativa se puede considerar una noción relativamente simple, habilita un tratamiento matemático preciso.

\footnotetext{
Mapas de competencias genéricas del bachillerato. Rafael Morales, Morales Gamboa, González Navarro y Reyes Sánchez.

Página 5 de 27
} 
En contraste, otros autores entienden las competencias de manera más holística, como integraciones de sus elementos (Karampiperis et al., 2006; D. Sampson et al., 2007), que combinan (Pépiot et al., 2007), organizan (IMS Global Learning Consortium, 2002) o aplican (Ramírez \& Sánchez, 2012) para que el sujeto pueda atender el entorno y producir ciertos desempeños. Aún otros autores enfatizan la naturaleza de la competencia como desempeño (Cicortas et al., 2008; Paquette, 2007; Paquette et al., 2006) e incluso la distinguen de su definición o declaración (Paquette, 2007; Paquette et al., 2006; Ramírez \& Sánchez, 2012), la se puede extender para incluir relaciones con otras competencias y ejemplos de la competencia en la práctica (Stoof et al., 2007). La diferencia más importante entre concebir a una competencia como integración de componentes que produce desempeños, o simplemente como desempeños, es que en el primer caso se reconoce que no es directamente observable ni medible (Cicortas et al., 2008; Miranda et al., 2017), en tanto que en el segundo caso se concibe de manera contraria (García-Barriocanal et al., 2012).

De manera aún más holística, algunos autores conciben a una competencia como una característica de un individuo o grupo, necesaria para producir un desempeño (Vasconcelos et al., 2001) o subyacente al mismo, habilitándolo (Cicortas et al., 2008), en tanto que otros autores las identifican como capacidades de usar los elementos o aplicar los elementos que las componen (May \& Ossendorf, 2014; Różewski \& Małachowski, 2009), para atender a las demandas del entorno (D. G. Sampson, 2009), producir desempeños (García-Barriocanal et al., 2012) o actuar de manera efectiva y eficiente en el entorno (Miranda et al., 2017).

\section{Componentes de competencias}

En lo que respecta los componentes de una competencia, los más referidos son conocimiento, habilidades y actitudes; en el caso de conocimiento, en algunas ocasiones se hace referencia, más específicamente, a conocimiento conceptual y conocimiento procedimental, en tanto que en el caso de las habilidades, se desglosan en ocasiones en habilidades motoras, cognitivas, genéricas, sociales y metodológicas. Algunos autores incluyen solamente una habilidad en una competencia (Lefebvre et al., 2005; Nkambou et al., 1996; Paquette et al., 2006; Ramírez \& Sánchez, 2012), en tanto que otros autores ofrecen una variedad de elementos internos al individuo (ideas, valores y experiencia) o bien externos al mismo (información, contactos, conocimiento de otros, recursos externos y herramientas).

\section{Tipos de competencias}

En dieciocho de los veintiséis artículos se deja suficientemente claro el tipo de competencias que se puede modelar con su esquema de representación. De estos, trece artículos indican que su esquema de representación es aplicable a cualquier tipo de competencia (Biletska et al., 2010; Competency Data Working Group, 2008; El Asame \& Wakrim, 2018; García-Barriocanal et al., 2012; IMS Global Learning Consortium,

\footnotetext{
Mapas de competencias genéricas del bachillerato. Rafael Morales, Morales Gamboa, González Navarro y Reyes Sánchez.

Página 6 de 27
} 
2002; Karampiperis et al., 2006; Leenheer et al., 2010; May \& Ossendorf, 2014; Różewski \& Małachowski, 2009; D. Sampson et al., 2007; D. G. Sampson, 2009; Stoof et al., 2007; Wang \& Wang, 2018).

Entre los otros cinco donde es claro el ámbito de aplicación de su esquema de representación, (Miranda et al., 2017) se enfoca a competencias profesionales, del ámbito laboral, en tanto que (Ramírez \& Sánchez, 2012) establece que se esquema aplica a competencias asociadas con un dominio de conocimiento; esto es, no para competencias genéricas a varios dominios. En (Lefebvre et al., 2005) se señala que se busca representar competencias específicas y genéricas, entiendo las primeras como aquellas directamente relacionadas con la ejecución de una tarea, en tanto que las segundas tienen un sentido más disciplinar y de uso más generalizado, como las modeladas por (Ramírez \& Sánchez, 2012). En cambio, en (Cicortas et al., 2008) se modelan lo que se denominan como 'seis competencias genéricas... competencia de aprendizaje, competencia ética, competencia comunicativa y social, competencia de desarrollo, competencia organizacional y social, y competencia de internacionalización'. Finalmente, (Pépiot et al., 2007) extiende el ámbito de representación a competencias de individuos, de unidades de trabajo y de organizaciones completas.

En cuatro de los artículos se presenta un campo de aplicación específico, al menos para ilustrar el potencial de su esquema de representación: competencias del lenguaje (Karampiperis et al., 2006), de toma de decisiones para la solución de problemas (Yu \& Zhang, 1990), de manejo de una bomba de infusión de Baxter por enfermeras (Nkambou et al., 1996), y de enfermería en general (Sitthisak et al., 2007).

\section{Relación con el contexto}

En lo referente a la relación de las competencias con su contexto de ejecución, varios autores consideran el contexto como parte de la definición de una competencia (Cicortas et al., 2008; Karampiperis et al., 2006; Ramírez \& Sánchez, 2012; D. Sampson et al., 2007; Yu \& Zhang, 1990). En (D. Sampson \& Fytros, 2008) se hace incluso una crítica a los estándares (Competency Data Working Group, 2008; IMS Global Learning Consortium, 2002) por no incluir mecanismos para la inclusión del contexto en la definición de una competencia. Asimismo, en (Pépiot et al., 2007) una competencia se relaciona directamente con el manejo de recursos (materiales e inmateriales) en un contexto.

En cambio, en (Paquette, 2007; Paquette et al., 2006) la descripción del contexto en la definición de una competencia es necesario solamente 'si se necesita más precisión, se puede agregar un grado de desempeño especificando el contexto situacional donde se va a aplicar la habilidad cognitiva: en contextos familiares o nuevos, de manera persistente o esporádica, en situaciones simples o complejas, etc.'.

Finalmente, otros autores comentan que se puede definir una competencia independientemente del contexto, incluso a pesar de que se necesita un contexto para

\footnotetext{
Mapas de competencias genéricas del bachillerato. Rafael Morales, Morales Gamboa, González Navarro y Reyes Sánchez.

Página 7 de 27
} 
demostrar la competencia (García-Barriocanal et al., 2012). Para (Miranda et al., 2017), la definición de una competencia implica necesariamente la generalización de los contextos en los cuales es pertinente.

\section{Relaciones entre competencias}

No todos los autores han considerado la representación de relaciones entre competencias. Específicamente, de los veintiséis artículos revisados, en cuatro de ellos no se menciona la posibilidad de representar relaciones entre competencias. Entre el resto de los autores, las relaciones entre competencias más comúnmente consideradas han sido las clásicas incluye/parte-de y subsumeles-un, comunes en el ámbito de la informática, ontología y matemáticas, entre otras disciplinas (García-Barriocanal et al., 2012; Karampiperis et al., 2006; Leenheer et al., 2010; Miranda et al., 2017; Pépiot et al., 2007; Ramírez \& Sánchez, 2012; Różewski \& Małachowski, 2009; D. Sampson et al., 2007; Vasconcelos et al., 2001). En el caso de (Vasconcelos et al., 2001), la relación incluye/parte-de se puede dar también entre los componentes de una competencia, en tanto que (Karampiperis et al., 2006; D. Sampson et al., 2007) consideran que cada subcompetencia - parte-de una competencia- debe tener un peso específico (importancia relativa) en el contexto de la competencia que la incluye.

Dos casos particularmente interesantes son los de (García-Barriocanal et al., 2012, p. 201) y (Miranda et al., 2017), porque en el primero se distingue entre la definición general de una competencia y el estado de la misma en cada individuo, estableciéndose una relación de subsume/es-un entre ellas, en tanto que en el segundo la relación subsume/es un es implícita entre competencias y se deriva de la relación entre una situación general, correspondiente a la definición de una competencia, y los contexto específicos en los cuales una competencia se desarrolla y se evalúa. Finalmente, en (Lefebvre et al., 2005) se añade una relación de "analogía" entre competencias, poco clara pero relacionada con el fenómeno de transferencia, y comentan sobre otra relación de "desviación", en tanto que en (Leenheer et al., 2010) a los dos tipos de relaciones básicas se pueden añadir otras según el caso.

En otros casos, como en la recomendación (IMS Global Learning Consortium, 2002) y el estándar (Competency Data Working Group, 2008), las relaciones entre competencias no están incluidas específicamente en la relación entre competencias, si bien se pueden añadir haciendo uso de mecanismos disponibles en otros estándares como (IEEE, 2002). En (Sitthisak et al., 2007, p. 200) se comenta que se modelan relaciones, sin especificar cuáles, mientras que en (El Asame \& Wakrim, 2018; Stoof et al., 2007; Wang \& Wang, 2018) se comenta que se puede modelar cualquier tipo de relación.

En (Paquette, 2007; Paquette et al., 2006) no se representan relaciones entre competencias sino relaciones entre las habilidades (componentes de competencias) y en (D. G. Sampson, 2009) se modelan relaciones entre competencias y objetos de aprendizaje. Finalmente, en (Nkambou et al., 1996) los componentes de las competencias pueden estar conectados a través de relaciones de subsume/es-un,

Mapas de competencias genéricas del bachillerato. Rafael Morales, Morales Gamboa, González Navarro y Reyes Sánchez.

Página 8 de 27 
abstracción/concretización, incluye/parte-de, analogía y desviación; pero no explican las diferencias entre las relaciones subsume/es-un y abstracción/concretización, mientras que la relación de analogía parece tener el mismo propósito, y los mismos problemas, que en (Lefebvre et al., 2005). Las competencias pueden estar relacionadas por otro tipo de relaciones: prerrequisito, agregación, complementariedad y "pretexto", además de que las relaciones entre componentes pueden extenderse a las competencias: "un objetivo [competencia] también está vinculado a los medios necesarios (recursos pedagógicos) para su realización, a otros objetivos [competencias], a prerrequisitos de capacidades [componentes] y capacidades para la adquisición de las cuales puede contribuir' (Nkambou et al., 1996, pág. 92).

\section{Esquema de representación}

Se han usado relativamente pocos esquemas para la representación de competencias, considerando la variedad de esquemas disponibles en las ciencias de la computación y la inteligencia artificial (Brachman \& Levesque, 2004), si bien la colección se extiende considerablemente cuando se busca representar y dar seguimiento al desarrollo de las competencias (ver sección ).

Algunos trabajos usan un esquema de representación matemático - expresable computacionalmente - como es el caso de conjuntos y lógica difusa (Yu \& Zhang, 1990), conjuntos difusos (Wang \& Wang, 2018) o grafos (Ramírez \& Sánchez, 2012). Alternativamente, se han usado ontologías (Leenheer et al., 2010; Vasconcelos et al., 2001). En el caso de (Lefebvre et al., 2005) se usan ontologías de habilidades y conocimientos para definir competencias, competencias para definir pericia, y pericia para definir documentos, usuarios, roles y organizaciones. Cicortas et al. (2008) hacen uso de ontologías para representar tipos de competencias y jerarquías de competencias, mientras que en (Miranda et al., 2017) se describe el uso de la pila de tecnologías de la Web Semántica (Berners-Lee, 2000).

El esquema más utilizado parece ser XML (World Wide Web Consortium, 2016), del cual se hace uso para definir recomendaciones y estándares de interoperabilidad entre sistemas (Competency Data Working Group, 2008; HR Open Standards Consortium, 2018; IMS Global Learning Consortium, 2002), los cuales son discutidos y extendidos por diversos autores (El Asame \& Wakrim, 2018; Karampiperis et al., 2006; D. Sampson et al., 2007; D. Sampson \& Fytros, 2008; D. G. Sampson, 2009; Sitthisak et al., 2007).

Otros esquemas de representación incluyen taxonomías, como en (May \& Ossendorf, 2014), que utilizan taxonomías de conocimiento, afectos y habilidades físicas, y en (Paquette, 2007; Paquette et al., 2006), que combinan taxonomías de habilidades cognitivas con lo que llaman tipos de objetos: 'conceptos (o clases de objetos), procedimientos (o clases de acciones) y principios (o clases de afirmaciones, propiedades o reglas'.

Mapas de competencias genéricas del bachillerato. Rafael Morales, Morales Gamboa, González Navarro y Reyes Sánchez.

Página 9 de 27 
Finalmente, en algunos casos se utilizan otros esquemas, como una Red de Transición del Conocimiento del Currículo (Curriculum Knowledge Transition Network, CKTN) con tres capas: de objetivos (competencias), de capacidades (conocimientos, habilidades) y de recursos, con vínculos entre las tres capas (Nkambou et al., 1996); o un lenguaje gráficos basado en el Unified Modeling Language (UML) (Object Management Group, 2003), como es el caso de UECML (Unified Enterprise Competence Modelling Language), descrito en (Pépiot et al., 2007).

\section{Niveles de competencia}

Varios de los esquemas de representación descritos en la literatura no consideran incluir una descripción del nivel de desarrollo de una competencia como parte de la representación de ésta. Tal es el caso de la recomendación de IMS Global (IMS Global Learning Consortium, 2002) y del estándar de IEEE (Competency Data Working Group, 2008), entre otras propuestas (May \& Ossendorf, 2014; Miranda et al., 2017; Ramírez \& Sánchez, 2012). Algunos autores (D. G. Sampson, 2009; Sitthisak et al., 2007) consideran que los estándares para el intercambio de representaciones de competencias comentados arriba debieran incluir niveles de competencia, en tanto que en otros trabajos no queda claro si la representación de competencias considera sus niveles (Biletska et al., 2010; Cicortas et al., 2008; Leenheer et al., 2010; Vasconcelos et al., 2001). En algunos más, simplemente se deja como una tarea que se puede realizar con cierta facilidad (Paquette, 2007; Paquette et al., 2006).

Entre los demás artículos, los niveles de competencia son atendidos de maneras diversas. En (Nkambou et al., 1996) se consideran cuatro niveles conocimiento de los elementos de una competencia de una persona: no se tiene información (ignorancia de parte del sistema), no lo conoce, lo conoce parcialmente o lo conoce (completamente). Otros autores consideran también niveles de adquisición, conocimiento o desarrollo de los componentes de una competencia (García-Barriocanal et al., 2012; Pépiot et al., 2007; D. Sampson \& Fytros, 2008).

En el caso de (Yu \& Zhang, 1990), se comienza considerando dos niveles, se tiene o no se tiene, aunque posteriormente se trabaja un concepto más general de medida, así como el uso de conjuntos difusos (grados de adquisicón de elementos de la competencia), pero solamente de manera teórica. El trabajo posterior de (Wang \& Wang, 2018) que parte del trabajo inicial de (Yu \& Zhang, 1990) hace uso se conjuntos difusos. Otros autores consideran rangos de desarrollo de una competencia, entre un valor mínimo y un valor máximo (Karampiperis et al., 2006; Lefebvre et al., 2005; D. Sampson et al., 2007), en tanto que en (El Asame \& Wakrim, 2018) se consideran solamente cuatro niveles, pero no como escalas de desarrollo de la misma competencia; esto es, cada nivel de competencia se consitituye en una competencia diferente.

Finalmente, están los trabajos que dejan abierta la posibilidad de definir los niveles de competencia que se consideren pertinentes (Różewski \& Małachowski, 2009; Stoof et al., 2007).

Mapas de competencias genéricas del bachillerato. Rafael Morales, Morales Gamboa, González Navarro y Reyes Sánchez.

Página 10 de 27 


\section{Desarrollo de competencias}

Como se comentó previamente en la sección, los intereses que han guiado la construcción de esquemas han sido variados y solamente en algunos casos el interés ha estado en la representación del estado de competencias de una persona y en menos casos todavía ha habido interés en promover o dar seguimiento al desarrollo de competencias en una persona.

En (Yu \& Zhang, 1990), y posteriormente en (Różewski \& Małachowski, 2009; Wang \& Wang, 2018), en el ámbito organizaciónal, se estima el tiempo necesario y el costo correspondiente para llegar a las competencias objetivo a partir de las competencias actuales. En (Vasconcelos et al., 2001) se habla de un sistema para la gestión de competencias grupales (group memory system), de modo que sería posible identificar niveles de competencia y necesidades de desarrollo, pero no se explica con detalle.

En (Lefebvre et al., 2005), en el ámbito educativo, se utiliza la Zona de Desarrollo Próximo de Vygotsky ("Zone of Proximal Development", 2009) para ofrecer recursos a la medid y se comenta sobre el uso de reglas que aplican a través de las relaciones entre competencias para propagar creencias sobre niveles de competencia (por ejemplo, si se tiene un nivel de pericia en una competencia, se tiene en todas sus subcompetencias). En (Biletska et al., 2010) se comenta también el uso de reglas para actualizar la estimación de nivel de competencia de una persona.

Destaca en este rubro el trabajo de Nkambou et al. (1996), quienes presentan un modelo detallado del seguimiento de adquisición de atributos de competencias por los estudiantes, de la adqusición de competencias completas, de recursos educativos dominados y de comprensión de las relaciones entre algunos de ellos. Básicamente, se modela la adquisición de todas las partes y construyen a partir de eso, y la propagación de creencias a través de las relaciones entre competencias se implementa mediante reglas asociadas a los tipos de relaciones.

\section{Marco de referencia}

El objetivo general del proyecto cuya primera etapa aquí se presenta es la construcción de soporte tecnológico para dar seguimiento al desarrollo por los estudiantes de todas y cada una de las competencias de un programa de estudios en línea. Se ha escogido como primera aproximación a este objetivo el seguimiento del desarrollo de las competencias genéricas del marco general común del Sistema Nacional de Bachillerato de México (Secretaría de Educación Pública, 2008) por dos razones principales: la cobertura nacional del programa de estudios, que amplía las posibilidades de evaluación y uso del desarrollo tecnológico, y la naturaleza transversal de las competencias genéricas, que dificulta estimar su desarrollo en la información ofrecida en el certificado de estudios de bachillerato.

En términos de la arquitectura típica de un sistema tutor inteligente, el objetivo del proyecto consiste en el desarrollo de un subsistema de modelado del estudiante (Elsom-

Mapas de competencias genéricas del bachillerato. Rafael Morales, Morales Gamboa, González Navarro y Reyes Sánchez. 
Cook, 1993; Holt et al., 1994)), el cual a su vez demanda la construcción de un modelo del dominio de conocimiento objeto de estudio por los estudiantes y un esquema de construcción del primero a partir del segundo. En nuestro caso, hemos optado por un esquema de sobrecapa (Holt et al., 1994; VanLehn, 1988), en el cual un modelo de un estudiante se construye como una colección de "creencias", una por elemento del dominio, que representa una estimación del grado de apropiación de cada elemento por el estudiante. Así, la construcción de un modelo de competencias del estudiante demanda la construcción de un modelo de las competencias a ser desarrolladas por el estudiante a lo largo de sus estudios, así como de un mecanismo de adscripción de una creencia por competencia como estimación del desarrollo de esa competencia por el estudiante.

Un primer aspecto a considerar en la construcción del modelo de las competencias consistió en acordar lo que era una competencia. Así, con base en lecturas previas (McClelland, 1973; Perrenoud, 2008) y la revisión del estado del arte (Cicortas et al., 2008; Miranda et al., 2017), se define competencia como

La capacidad de una persona para actuar de manera efectiva y confiable para el logro de propósitos similares en cierto tipo de situaciones.

Una competencia se observa solamente a través de los desempeños de las personas. Sin embargo, con propósitos teóricos, explicativos, una competencia se modela como la integración y movilización de una colección de componentes internos a la persona; específicamente, en concordancia con el estado del arte, se identificaron conocimiento, habilidades y actitudes como componentes de una competencia, a los cuales se añadieron valores como el cuarto tipo de elemento de una competencia, como atractores del comportamiento de la persona que ha desarrollado la competencia. Asimismo, en concordancia con varios de los autores revisados en la sección anterior, se ha adoptado un enfoque holístico, en el sentido de que una competencia, como integración de sus componentes, es mayor que la suma de sus partes y no puede ser evaluada parte por parte.

Si bien el trabajo que aquí se presenta está enfocado en competencias genéricas del bachillerato en México, consideramos que la definición arriba establecida y los desarrollos que se describen posteriomente son aplicables a cualquier tipo de competencia, al menos en el ámbito educativo.

\section{Relaciones y contexto}

Se han adoptado las relaciones comunes de incluye/parte-de y subsumeles-un (esta última descrita también como una relación generaliza/especializa) entre competencias (Chan et al., 2010), con la siguiente semántica:

1. Los componentes de una competencia incluida (subcompetencia) son parte de

Mapas de competencias genéricas del bachillerato. Rafael Morales, Morales Gamboa, González Navarro y Reyes Sánchez.

Página 12 de 27 
los componentes de la competencia incluyente (supercompetencia).

2. Una supercompetencia tiene al menos un componente más (conocimientos, habilidades, actitudes o valores) que cualquiera de sus subcompetencias.

3. Una competencia subsumida (que especializa) hereda los componentes de la competencia que la subsume (generaliza).

4. Una competencia subsumida incluye la estructura de subcompetencias de la competencia que la generaliza, o especializaciones de éstas.

5. Una competencia subsumida incluye al menos un componente o una subcompetencia más que la competencia que especializa.

Si bien es posible considerar competencias que especializan a más de una competencia (herencia múltiple) esto no ha sido considerado hasta ahora como parte del proyecto.

Por otra parte, se ha comentado arriba la estrecha relación que existe entre la competencia, como capacidad de acción, y las situaciones en las cuáles tiene lugar. Una competencia en la línea de 'usar un procesador de documentos para elaborar o modificar un documento' tiene sentido en una situación en la cual existe un dispositivo digital, como una computadora de escritorio o portatil, conectado a alguna fuente de poder interna (batería) o externa (servicio de electricidad), accesible de manera conveniente para el usuario y en la cual se encuentra instalada una aplicación de tipo procesador de documentos, etc. Esta relación entre una competencia y el contexto de ejecución de la misma ha llevado a varios autores a incluir el contexto como parte de la definición de la competencia, como se observó en la revisión del estado del arte, y esa misma decisión se tomó en las primeras etapas del proyecto que aquí se describe (Chan et al., 2010).

Sin embargo, la observación de que las relaciones entre competencias reflejan relaciones entre situaciones (contextos), así como la observación de que los componentes de la competencia definen en buena medida el contexto de ejecución de la competencia - por ejemplo, conocimientos y habilidades para operar un procesador de documentos implican la existencia de un procesador de documentos en el contextonos llevaron a no darle un tratamiento formal al contexto e introducirlo solamente como parte de la descripción textual de la competencia.

\section{Esquema de representación}

Como se comentó previamente, una competencia se modela como la integración de cuatro tipos de elementos: conocimientos, habilidades, actitudes y valores. Además, una competencia puede incluir (sub)competencias, así como generalizar o especializar a otra competencia, con las condiciones establecidas previamente.

En la práctica, los mapas de las competencias se han construido usando la herramienta CmapTools (cmap.ihmc.us); esto es, representados como mapas conceptuales y usando las etiquetas 'tiene', 'incluye' y 'es un' para denotar relaciones entre competencias y

Mapas de competencias genéricas del bachillerato. Rafael Morales, Morales Gamboa, González Navarro y Reyes Sánchez.

Página 13 de 27 
elementos, competencias y subcompetencias, y competencias y especializaciones de éstas. respectivamente.

Por otra parte, se ha usado un base de datos relacional para almacenar tanto los mapas de competencias como las redes bayesianas dinámicas construídas con base en ellos, con las cuales se implementa el modelo de sobrecapa usando distribuciones de probabilidad de tres niveles de competencia (bajo, medio y alto) para representar creencias sobre el estado de las competencias de los estudiantes (Morales Gamboa \& Sucar, en revisión; Rodríguez Yépez \& Morales Gamboa, 2019).

\section{Metodología}

Como se comentó en la Introducción, el análisis de las competencias genéricas del Sistema Nacional de Bachillerato que aquí se presenta estuvo motivado por la construcción de una representación computacional de las mismas que pudiera ser aprovechado por un sistema computacional para dar seguimiento al desarrollo de las competencias de los estudiantes usando técnicas de inteligencia artificial. De acuerdo con el modelo de competencia seleccionado y las relaciones de interés entre competencias, descritos en la sección anterior, el énfasis del ánalisis estuvo en la identificación de competencias (un hacer, generalmente relacionado con un verbo), sus atributos (conocimientos, habilidades, actitudes y valores) y sus relaciones de tipo incluye/parte-de y subsume/es-un con otras competencias. La capacidad de los sistemas computacionales de alojar y procesar grandes cantidades de información y con un alto nivel de detalle nos permitía desglosar tanto como fuera necesario las competencias oficiales —orientadas a ser leídas y comprendidas por seres humanos - siguiendo estos dos tipos de relaciones.

Como se ha comentado en un publicación previa (Medina Flores \& Morales Gamboa, 2017), el análisis de cada una de las competencias genéricas se realizó inicialmente de manera individual y se expusieron al grupo el proceso, los hallazgos y los resultados, mismos que fueron discutidos, modificados grupalmente $\mathrm{y}$, con cierta frecuencia, revisados de manera individual como inicio de una segunda ronda de análisis y revisión.

El análisis consistió, de manera general, en

- incluir las macrocompetencias como parte del análisis;

- identificar las capacidades incluidas en la descripción de la competencia y sus atributos;

- determinar cuáles capacidades hacían sentido más como competencias o más como habilidades;

- identificar los demás elementos de la competencia (conocimientos, actitudes y valores) implícitos en el texto;

- establecer relaciones entre todos los elementos identificados y catalogados.

Mapas de competencias genéricas del bachillerato. Rafael Morales, Morales Gamboa, González Navarro y Reyes Sánchez.

Página 14 de 27 


\section{Análisis}

En el Acuerdo número 444, publicado en el Diario Oficial de la Federación, la Secretaría de Educación Pública de México estableció, organizadas en seis grandes macrocompetencias, las competencias genéricas — esto es, no adscritas a una disciplina en particular y, por consiguiente, genéricas a ellas- que 'han de articular y dar identidad' a la educación media superior en nuestro país, (Secretaría de Educación Pública, 2008), las cuales se presenta en la Tabla 1. Cada competencia, a su vez, se desglosa en una serie de atributos que denotan capacidades de hacer — 'enfrenta', 'identifica', 'elige' y 'analiza', entre otros - los cuales pueden ser interpretados como habilidades o como subcompetencias. Otros elementos de la competencia, como conocimientos, actitudes y valores, se describen de manera implícita en cada atributo. Por ejemplo, la competencia 1 tiene como atributo

Identifica sus emociones, las maneja de manera constructiva y reconoce la necesidad de solicitar apoyo ante una situación que lo rebase. [Énfasis de los autores]

el cual incluye las capacidades de 'identificar emociones', 'manejar emociones de manera constructiva' y 'reconocer la necesidad de solicitar apoyo', en tanto que sugiere una actitud 'constructiva'.

Tabla 1. Competencias genéricas (genéricas) del Sistema Nacional de Bachillerato de México. Cita textual, reorganizada en tabla, del texto del Acuerdo número 444 (Secretaría de Educación Pública, 2008).

\begin{tabular}{ll}
\hline Macrocompetencias & Competencias \\
\hline Se autodetermina y cuida de sí. & $\begin{array}{l}\text { Se conoce y valora a sí mismo y aborda } \\
\text { problemas y retos teniendo en cuenta los } \\
\text { objetivos que persigue. }\end{array}$ \\
2. & Es sensible al arte y participa en la apreciad
\end{tabular}

2. Es sensible al arte y participa en la apreciación e interpretación de sus expresiones en distintos géneros.

Se expresa y comunica.

3. Elige y practica estilos de vida saludables.

4. Escucha, interpreta y emite mensajes pertinentes en distintos contextos mediante la utilización de medios, códigos y herramientas apropiados.

Piensa crítica y reflexivamente. 5. Desarrolla innovaciones y propone soluciones a problemas a partir de métodos establecidos.

6. Sustenta una postura personal sobre temas de interés y relevancia general, considerando otros puntos de vista de manera crítica y reflexiva.

Aprende de forma autónoma.

7. Aprende por iniciativa e interés propio a lo largo de la vida.

\footnotetext{
Mapas de competencias genéricas del bachillerato. Rafael Morales, Morales Gamboa, González Navarro y Reyes Sánchez. 
Trabaja en forma colaborativa.

Participa con responsabilidad en la sociedad.
8. Participa y colabora de manera efectiva en equipos diversos.

9. Participa con una conciencia cívica y ética en la vida de su comunidad, región, México y el mundo.

10. Mantiene una actitud respetuosa hacia la interculturalidad y la diversidad de creencias, valores, ideas y prácticas sociales.

11. Contribuye al desarrollo sustentable de manera crítica, con acciones responsables.

A manera de ejemplo, se incluye a continuación la defición completa de la competencia genérica 1 y se presenta el mapa correspondiente en la .

\section{Se autodetermina y cuida de sí}

1. Se conoce y valora a sí mismo y aborda problemas y retos teniendo en cuenta los objetivos que persigue.

Atributos:

- Enfrenta las dificultades que se le presentan y es consciente de sus valores, fortalezas y debilidades.

- Identifica sus emociones, las maneja de manera constructiva y reconoce la necesidad de solicitar apoyo ante una situación que lo rebase.

- Elige alternativas y cursos de acción con base en criterios sustentados y en el marco de un proyecto de vida.

- Analiza críticamente los factores que influyen en su toma de decisiones.

- Asume las consecuencias de sus comportamientos y decisiones.

- Administra los recursos disponibles teniendo en cuenta las restricciones para el logro de sus metas. 


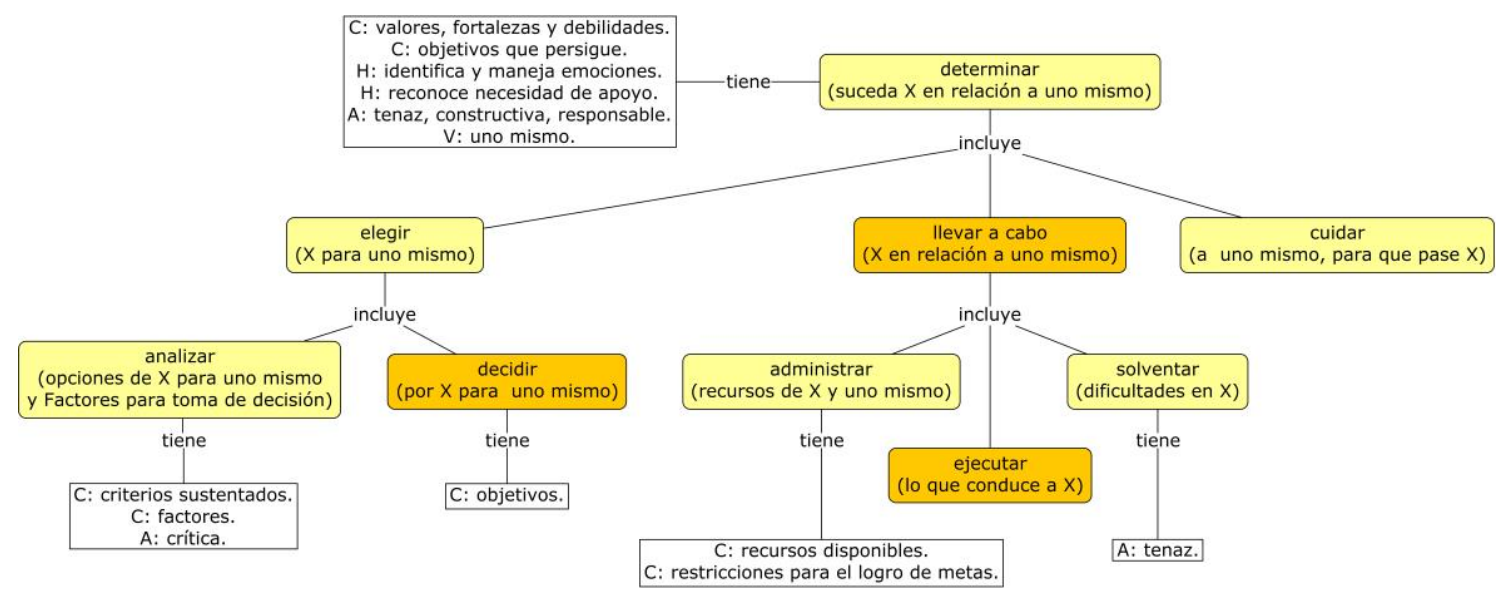

Figura 2. Mapa correspondiente a la competencia genérica 1.

En este caso se observó que la macrocompetencia está compuesta por dos competencias, autodeterminarse y cuidar de sí. Se observó también que la segunda es condición para lograr la primera, de modo que se incluyó como subcompetencia de ésta. Se opta por reemplazar la capacidad de enfrentar dificultades por solventarlas, ya que la segunda implica además encontrar una solución, con actitud tenaz, y se interpretó 'es consciente' como 'conoce', de modo que valores, fortalezas y debilidades se interpretan como atributos de conocimiento de la competencia. Se identifica analizar factores como condición para poder elegir alternativas, de modo que la primera, con un atributo de actitud crítica, se interpreta como subcompetencia de la segunda y se complementa con una competencia adicional, decidir. Se introduce la competencia llevar a cabo, encargada de hacer la elección realidad, que incluye a administrar (recursos) y solventar (dificultades) e incluye una competencia adicional, ejecutar. Las capacidades de ámbito interno, cognitivas y afectivas, se interpretan generalmente como habilidades, como es identificar emociones propias, manejarlas adecuadamente y reconocer (el estado interno de) necesidad de apoyo.

En el mapa resultante () se marcan - en color naranja - las competencias que no fueron extraídas directamente de la descripción oficial de la competencia genérica, pero se consideraron implícitas o fueron añadidas para dar coherencia al mapa.

Los mapas de todas las competencias se integraron en un único mapa de competencias genéricas, al cual se añadieron competencias — marcadas en verde - que generalizaban competencias similares en mapas distintos. Por ejemplo, en la competencia número dos (Tabla 1) se identificó la competencia de practicar el arte con algún propósito similar a la competencia practicar un estilo de vida para ser saludable identificada en la competencia número tres, por lo que se introduje la competencia más general de practicar algo con un cierto propósito, que subsume a las dos anteriores. En otros casos, se identificaron relaciones directas de generalización/especialización entre competencias en los mapas, como es el caso de la competencia cuidar a uno mismo, en el mapa de la competencia número uno, y cuidar a uno mismo para ser saludable, en el mapa de la competencia número tres.

Mapas de competencias genéricas del bachillerato. Rafael Morales, Morales Gamboa, González Navarro y Reyes Sánchez.

Página 17 de 27 


\section{Resultados}

El mapa completo de competencias genéricas está compuesto por 116 competencias, de las cuales 93 fueron recuperadas de las descripciones de las competencias genéricas del bachillerato, 15 fueron añadidas a los mapas individuales y 8 fueron añadidas directamente en el mapa completo. Solamente el 36\% (42) de las competencias cuentan con atributos. El mismo mapa cuenta con un total de 141 enlaces, de los cuales 87 son de tipo incluye/parte-de y 54 son de tipo subsume/es-un.

El conjunto de los once mapas por competencia genérica del bachillerato, así como el mapa integrado, se encuentran disponibles en formato CXL (Concept Mapping Extensible Language) de CmapTools (IHMC, s/f) en GitLab (https://gitlab.com/rmorales/macomba).

\section{Etiquetado de cursos}

Como se comentó previamente, el objetivo de la construcción del mapa de competencias genéricas es dar soporte al seguimiento fino del desarrollo de esas competencias por los estudiantes de bachillerato con base en la evidencia que se recolecte al respecto, la cual podría provenir de cualquier actividad realizada por los estudiantes en las que se demuestre la aplicación de las competencias genéricas —en principio, casi cualquier actividad realizada en el medio digital. Sin embargo, es de esperarse que la fuente principal de evidencias sean los cursos del programa de bachillerato, para lo cual es necesario establecer vínculos entre las competencias en el mapa y las actividades en los cursos.

Se procedió entonces a realizar un primer ejercicio de etiquetado de actividades en cursos del programa de Bachillerato General por Áreas Interdisciplinarias (BGAI) del Sistema de Universidad Virtual de la Universidad de Guadalajara (Sistema de Universidad Virtual, 2021) usando las competencias en el mapa, con dos fines:

1. Vincular el mapa de competencias con el diseño instruccional de dichos cursos.

2. Conocer el grado en que las competencias genéricas del bachillerato están presentes en el diseño instruccional de los cursos del BGAI.

Se tomaron diez cursos de muestra, de los veintiuno que integra la ruta de formación del BGAI. La muestra los cursos etiquetados, los cuales correspondieron al ciclo escolar 2019A (enero-julio).

\footnotetext{
Mapas de competencias genéricas del bachillerato. Rafael Morales, Morales Gamboa, González Navarro y Reyes Sánchez. 
Tabla 2: Cursos del Bachillerato General por Áreas Interdisciplinarias que fueron etiquetados con competencias (negritas) en el marco del área de formación básica del programa de estudios.

\begin{tabular}{|c|c|c|c|}
\hline Primer ciclo & Segundo ciclo & Tercer ciclo & Cuarto ciclo \\
\hline Matemática y vida & Matemática y ciencia & Precálculo & Matemática avanzada \\
\hline cotidiana & 10 créditos & 5 créditos & 5 créditos \\
\hline $\begin{array}{l}\text { Comprensión y } \\
\text { expresión verbal } \\
8 \text { créditos }\end{array}$ & $\begin{array}{l}\text { Descripción, análisis } \\
\text { y argumento } \\
9 \text { créditos }\end{array}$ & $\begin{array}{l}\text { Corrección de estilo y } \\
\text { crítica propositiva } \\
10 \text { créditos }\end{array}$ & $\begin{array}{l}\text { Geografía para la } \\
\text { sustentabilidad } \\
10 \text { créditos }\end{array}$ \\
\hline $\begin{array}{l}\text { Autodeterminación } \\
\text { y aprendizaje }\end{array}$ & $\begin{array}{l}\text { Arte y cultura } \\
\text { regional } \\
9 \text { créditos }\end{array}$ & $\begin{array}{l}\text { Actividad física y } \\
\text { desarrollo deportivo } \\
6 \text { créditos }\end{array}$ & $\begin{array}{l}\text { Ética y política } \\
10 \text { créditos }\end{array}$ \\
\hline 15 créditos & Vida saludable & Lengua extranjera I & $\begin{array}{l}\text { Lengua extranjera II } \\
15 \text { créditos }\end{array}$ \\
\hline $\begin{array}{l}\text { Tecnologías de la } \\
\text { información }\end{array}$ & 11 créditos & 13 créditos & Perspectiva de género \\
\hline 12 créditos & $\begin{array}{l}\text { Química } \\
14 \text { créditos }\end{array}$ & $\begin{array}{l}\text { Biología } \\
14 \text { créditos }\end{array}$ & $\begin{array}{l}\text { Obligatoria sin } \\
\text { créditos }\end{array}$ \\
\hline $\begin{array}{l}\text { Física y } \\
\text { conocimiento } \\
\text { científico } \\
19 \text { créditos }\end{array}$ & $\begin{array}{l}\text { Identidad y } \\
\text { ciudadanía } \\
15 \text { créditos }\end{array}$ & & \\
\hline
\end{tabular}

Para dar inicio con el etiquetado por competencias genéricas, lo primero que se pensó fue en cómo se podría avanzar en el etiquetado sin perder de vista cada uno de los elementos involucrados en el proceso: el diseño instruccional de los cursos, las competencias genéricas presentadas en el Acuerdo 444 (Secretaría de Educación Pública, 2008), y el mapa completo de competencias genéricas descrito en la sección anterior. Se diseñó entonces una matriz para concentrar el etiquetado con las columnas que se muestran en la Tabla 3, que corresponden, por un lado, a los elementos que se contemplan en el modelo de diseño instruccional del Sistema de Universidad Virtual para todos sus cursos, que se dividen en unidades, éstas en actividades y cada una de las actividades incluye el objetivo de aprendizaje, una introducción, las instrucciones, recomendaciones y los criterios de evaluación. Por otro lado, se incluye el fragmento de texto donde se identifica la presencia de una competencia genérica, la competencia correspondiente en el Acuerdo, los atributos específicos de la competencia identificados en el fragmento de texto y la(s) competencia(s) directamente relacionada(s) en el mapa.

El proceso de etiquetado constó de tres fases, las cuales se describen a continuación.

\footnotetext{
Mapas de competencias genéricas del bachillerato. Rafael Morales, Morales Gamboa, González Navarro y Reyes Sánchez.

Página 19 de 27
} 
Tabla 3: Matriz de etiquetado de cursos. Las columnas corresponden a los rubros identificados en el análisis y los renglones a los distintos elementos del diseño instruciconal de un curso.

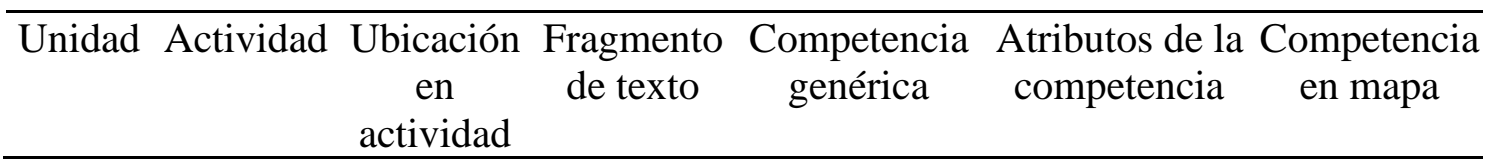

Fase 1

En esta primera fase se transcribió el diseño instruccional directamente en las primeras cuatro columnas de la matriz, a fin de identificar las acciones a realizar por el estudiante.

\section{Fase 2}

La segunda fase del etiquetado consistió en consultar la descripción de las competencias genéricas y sus atributos en el Acuerdo con la finalidad de relacionar cada una de los componentes de las actividades indicadas en el curso con las competencias genéricas y sus atributos. Se procedió entonces a recortar el texto en la tercera columna para dejar solamente los fragmentos relevantes, incluir la competencia genérica identificada en dichos fragmentos y, posteriormente, añadir los atributos a los que abonaban directamente. Así se procedió, sucesivamente, para cada elemento de las actividades en el diseño instruccional hasta concluir el curso.

\section{Fase 3}

En esta tercera fase se usó el etiquetado previo por competencias genéricas y atributos del Acuerdo para guiar la búsqueda de competencias más específicas en el mapa de competencias y llenar así la última columna de la matriz.

\section{Reflexiones}

Se presentan a continuación algunas reflexiones sobre la experiencia de etiquetado de cursos del BGAI.

- La actividad de etiquetado requiere de la concentración que permita la reflexión para lograr comprender qué es lo que el estudiante debe hacer, en qué contexto, qué producto obtendrá, con qué enfoque, como lo evidenciará o representará; esto para cada uno de los fragmentos de las actividades, unidades y cursos del BGAI.

- Es importante leer todo el curso antes de iniciar el etiquetado, con el objetivo de comprender mejor cada fragmento de texto.

- Se observan competencias genéricas en todos los elementos del diseño instruccional, si bien no existe una representación equitativa de todas ellas. Sería

Mapas de competencias genéricas del bachillerato. Rafael Morales, Morales Gamboa, González Navarro y Reyes Sánchez.

Página 20 de 27 
necesario etiquetar los cursos restantes del BGAI para poder emitir un juicio al respecto.

- Las unidades, objetivos y actividades de los cursos se relacionan con frecuencia, al menos potencialmente, con más de una competencia genérica del Acuerdo. Sin embargo, su redacción tiende a ser demasiado vaga, pues solamente se hace referencia a la acción genérica (por ejemplo, 'Realizarás un resumen' o 'Diseñarás'), lo que dificulta realizar un etiquetado más preciso porque no está clara la competencia que se busca desarrollar.

- En ocasiones resultó fácil identificar la competencia genérica del Acuerdo correspondiente a un elemento del diseño, pero al mismo tiempo se identificó la ausencia de atributos pertinentes en la descripción en el Acuerdo.

- Se idenficaron algunas inconsistencias en la construcción del mapa de competencias, específicamente en lo referente a la clasificación de las competencias genéricias del Acuerdo y sus atributos como competencias o como habilidades. Esto es, se identificaron casos similares con decisiones diferentes, que fueron corregidos en el mapa.

- El mapa de competencias ayudó a visualizar e identificar con mayor claridad y precisión la competencia genérica identificada en el texto, así como el alcance de la misma en el proceso de aprendizaje del estudiante. Con frecuencia, fue la identificación de la competencia en el mapa lo que permitió relacionarla el elemento de diseño con las competencias del Acuerdo y sus atributos, así como corregir selecciones previas de ellos.

Las competencias genéricas establecidas en el Acuerdo 444 que aparecieron con más frecuencia a lo largo del etiquetado son, en primer lugar, la competencia 4, Escucha, interpreta y emite mensajes pertinentes en distintos contextos mediante la utilización de medios, códigos y herramientas apropiados (ver Tabla 1), que se relaciona directamente con la lectura y comprensión de las instrucciones de cada actividad y la elaboración del producto correspondiente, así como con la participación en foros e incluso el trabajo colaborativo.

La segunda competencia genérica más frecuente, que va de la mano con la anterior, es la competencia 5, Desarrolla innovaciones y propone soluciones a problemas a partir de métodos establecidos. Esto se debe, tal vez, a que los modelo de diseño instruccional, tanto del Sistema de Universidad Virtual como el propuesto por Reforma Integral de la Educación Media Superior de México fueron diseñados bajo el enfoque constructivista.

La visión más detallada, desde la perspectiva del mapa de competencias genéricas, se puede observar en la, donde se presenta la frecuencia de identificación de las competencias contenidas en el mapa de competencias genéricas en los diez cursos revisados. De manera general, se identificaron 89 de las 116 competencias en el mapa, lo cual sugiere una buena cobertura de las competencias genéricas en los cursos

Mapas de competencias genéricas del bachillerato. Rafael Morales, Morales Gamboa, González Navarro y Reyes Sánchez. 


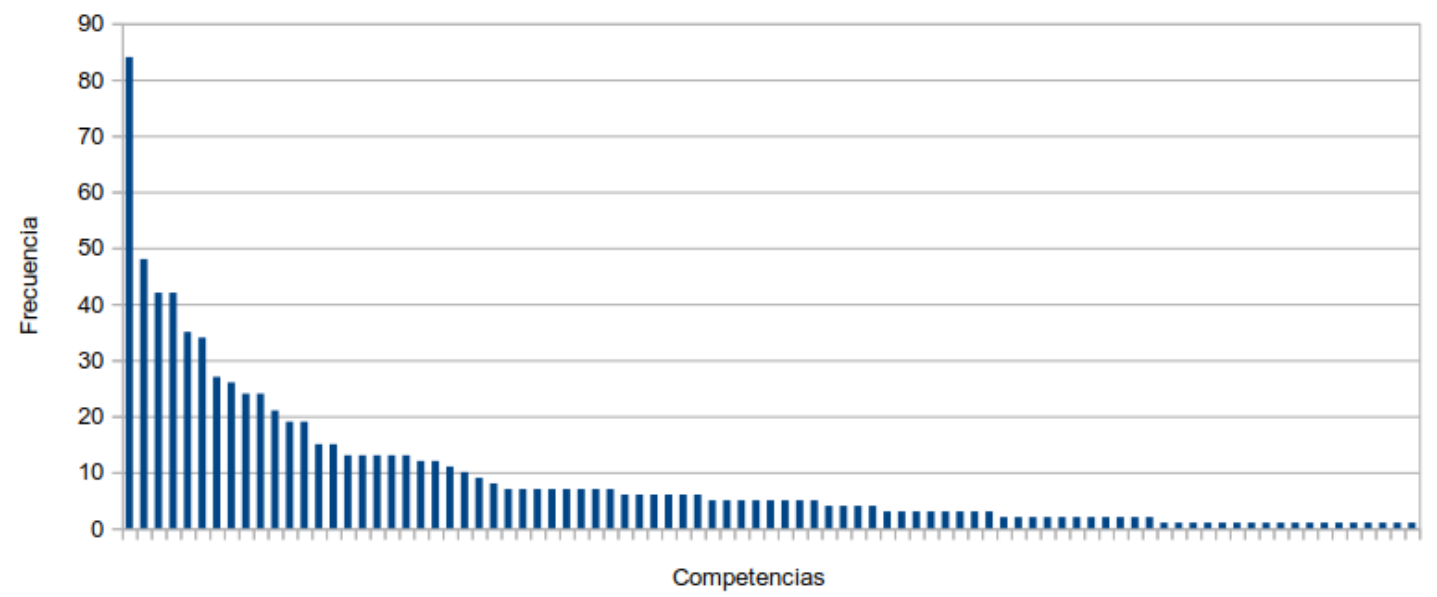

Figura 3: Frecuencia de identificación de las competencias en el mapa de competencias genéricas.

revisados. Sin embargo, 45 de las competencias aparecen a lo más una vez en diez cursos y solamente 24 de las competencias aparecen por lo menos diez veces en el total de cursos revisados.

\section{Conclusiones}

La revisión del estado del arte en la representación computacional de competencias ha permitido confirmar la alineación de nuestro proyecto de diseño e implementación de un entorno virtual inteligente para dar seguimiento al desarrollo de competencias por los estudiantes con la concepción general de competencias y su modelado en términos de conocimientos, habilidades, actitudes y valores, así como el uso de las relaciones básicas de subsume/es-un e incluye/parte-de para conectar competencias. La innovación introducida por nuestro proyecto está en su aplicación en un contexto real, el Sistema Nacional de Bachillerato de México, partiendo de las competencias genéricas establecidas formalmente para dicho sistema, su uso para la identificación de competencias en cursos de bachillerato y el uso de técnicas de inteligencia artificial (redes bayesianas dinámicas) para dar seguimiento puntual al desarrollo de las competencias por los estudiantes.

El análisis de las once competencias genéricas establecidas para el Sistema Nacional de Bachillerato de México dio como resultado un mapa con 116 competencias y 141 enlaces, lo cual sugiere mucha vaguedad en la descripción oficial de las competencias, así como la posibilidad de un seguimiento mucho más fino del desarrollo de competencias por los estudiantes, lo cual fue confirmado por el etiqueta por competencias de los elementos del diseño instruccional de cursos del Bachillerato 
General por Áreas Interdisciplinarias del Sistema de Universidad Virtual de la Universidad de Guadalajara.

El mapa de competencias genéricas del bachillerato mexicano sienta las bases para trabajos futuros en el seguimiento del desarrollo de estas competencias por los estudiantes. Información que puede ser sumamente útil para los mismos estudiantes, porque les ofrecería una descripción detallada y justificada del estado de su aprendizaje que, a su vez, les permitiría tomar decisiones sobre estrategias a seguir para mejorarlo; para los docentes, porque les permitiría conocer las condiciones de las competencias de sus estudiantes en cualquier momento y, con base en esa información, ofrecer atención personalizada a sus necesidades de formación, así como incidir de manera puntual en el diseño de los cursos y el etiquetado por competencias; para la administración universitaria, ofrecería información clave para evaluar programas educativos, identificar necesidades de rediseño de cursos y ofrecer microcredencialización por competencias, entre otras acciones.

Asimismo, los resultados del proyecto a la fecha ofrecen varias oportunidades para trabajo futuro, entre las que se encuentran realizar un tratamiento más formal de los atributos de las competencias, que actualmente se agrupan textualmente en un solo nodo, incrementar significativamente los atributos de las competencias en el mapa, pues la mayoría carece de ellos, y distinguir entre lo que se podría denominar la descripción formal de las competencias (todavía relativamente informal) y una descripción textual de las mismas que facilitaría su comprensión. CmapTools ha sido una excelente herramienta de trabajo y pudiera seguir siendo usada para facilitar la edición del mapa de competencias genéricas, pero la descripción formal del mismo debiera codificarse de acuerdo con un estándar internacional. Finalmente, una metodología similar, o incluso más formalizada, podría ser utilizada para generar mapas de competencias disciplinares para el bachillerato, así como mapas de competencias de otros programas de estudio o incluso mapas de competencias laborales.

Presentación del artículo: 17 de julio 2021

Fecha de aprobación: 24 de septiembre de 2021

Fecha de publicación: 30 de septiembre de 2021

Morales Gamboa, R., González Navarro, M.I. \& Reyes Sánchez, Y. (2021) Mapas de competencias genéricas del bachillerato. RED. Revista de educación a distancia, 21(67). http://dx.doi.org/10.6018/red.486531

\section{Financiación}

Este trabajo no ha recibido ninguna subvención específica de los organismos de financiación en los sectores públicos, comerciales o sin fines de lucro 


\section{Referencias}

Berners-Lee, T. (2000, diciembre 6). Semantic Web-XML2000. World Wide Web Consortium (W3C). https://www.w3.org/2000/Talks/1206-xml2k-tbl/Overview.html

Biletska, O., Biletskiy, Y., Li, H., \& Vovk, R. (2010). A semantic approach to expert system for e-Assessment of credentials and competencies. Expert Systems with Applications, 37, 7003-7014. https://doi.org/10.1016/j.eswa.2010.03.018

Brachman, R. J., \& Levesque, H. J. (2004). Knowledge Representation and Reasoning. Morgan Kaufmann.

Chan, M. E., González, S. C., \& Morales, R. (2010). A competency analyser as a knowledge-based approach for making e-learning more flexible and personalised. EDULEARN10 Proceedings CD. 2nd International Conference on Education and New Learning Technologies (Edulearn), 1607-1612. http://library.iated.org/view/CHAN2010ACO

Cicortas, A., Iordan, V., \& Naaji, A. (2008). Ontologies for Competence Description in Multi-agent Systems. Computational Methods and Intelligent Systems, 100-107. http://www.wseas.org/multimedia/books/2008/sofia/computational-methods-andintelligent-systems.pdf

Competency Data Working Group. (2008). 1484.20.1-2007 IEEE Standard for Learning Technology-Data Model for Reusable Competency Definitions (https://drive.google.com/drive/folders/0B1nfYUyald9QUmxkVURCcEs4WFE?usp =sharing). The Institute of Electrical and Electronics Engineers, Inc; Google Drive. http://ieeexplore.ieee.org/document/4445693/

El Asame, M., \& Wakrim, M. (2018). Towards a competency model: A review of the literature and the competency standards. Education and Information Technologies, 23(1), 225-236. https://doi.org/10.1007/s10639-017-9596-z

Elsom-Cook, M. (1993). Student modelling in intelligent tutoring systems. 7(3-4), 227240.

García-Barriocanal, E., Sicilia, M.-Á., \& Sánchez-Alonso, S. (2012). Computing with competencies: Modelling organizational capacities. Expert Systems with Applications, 39, 12310-12318. https://doi.org/10.1016/j.eswa.2012.02.194

Holt, P., Dubs, S., Jones, M., \& Greer, J. (1994). The State of Student Modelling. En J. Greer \& G. I. McCalla (Eds.), Student Modelling: The Key to Individualized Knowledge-Based Instruction (pp. 3-35). Springer Verlag. https://link.springer.com/chapter/10.1007/978-3-662-03037-0_1

HR Open Standards Consortium. (2018). HR Open Standards 4.1.1 Patch. HR Open Standards Consortium. https://www.hropenstandards.org

IEEE. (2002). 1484.12.1 Standard for Learning Object Metadata. IEEE.

IHMC. (s/f). CXL - Concept Mapping Extensible Language [XML Schema Definition]. CmapTools. Recuperado el 5 de julio de 2021, de https://cmap.ihmc.us/xml/CXL.html

IMS Global Learning Consortium. (2002). IMS Reusable Definition of Competency or Educational Objective Specification (Version 1.0 Final Specification; p. 11). IMS Global Learning Consortium, Inc.

Mapas de competencias genéricas del bachillerato. Rafael Morales, Morales Gamboa, González Navarro y Reyes Sánchez.

Página 24 de 27 
http://www.imsglobal.org/competencies/index.html

IMS Global Learning Consortium. (2003). Learning Design Specification. IMS Global Learning Consortium, Inc. http://www.imsglobal.org/learningdesign/

Karampiperis, P., Sampson, D., \& Fytros, D. (2006). Lifelong Competence Development: Towards a Common Metadata Model for Competencies Description The Case Study of Europass Language Passport. Proceedings of the Sixth International Conference on Advanced Learning Technologies (ICALT'06), 677-681. https://www.computer.org/csdl/proceedings/icalt/2006/2632/00/263200136-abs.html

Lee, J. F., \& Benati, A. G. (2009). Research and Perspectives on Processing Instruction. Walter de Gruyter.

Leenheer, P. D., Christiaens, S., \& Meersman, R. (2010). Business semantics management: A case study for competency-centric HRM. Computers in Industry, 61(8), 760-775. https://doi.org/10.1016/j.compind.2010.05.005

Lefebvre, B., Gauthier, G., Tadie, S., Duc, T., \& Achaba, H. (2005). Competence ontology for domain knowledge dissemination and retrieval. Applied Artificial Intelligence, 19(9-10), 845-859. https://doi.org/10.1080/08839510500234222

May, D., \& Ossendorf, P. (2014). ModellING competences: Developing a holistic competence model for engineering education. 2014 International Conference on Interactive Collaborative Learning (ICL), 877-894. https://doi.org/10.1109/ICL.2014.7017902

McClelland, D. C. (1973). Testing for competence rather than for "intelligence." American Psychologist, 28(1), 1-14. https://doi.org/10.1037/h0034092

Medina Flores, R., \& Morales Gamboa, R. (2017). Mapa de competencias genéricas del Acuerdo 444 de la RIEMS para entornos virtuales inteligentes basados en competencias. Memorias del 8o. Coloquio Nacional de Educación Media Superior a Distancia. Octavo Coloquio Nacional de Educación Media Superior a Distancia, León, Guanajuato.

Miranda, S., Orciuoli, F., Loia, V., \& Sampson, D. (2017). An ontology-based model for competence management. Data \& Knowledge Engineering, 107, 55-66. https://doi.org/10.1016/j.datak.2016.12.001

Morales Gamboa, R., \& Sucar, L. E. (2019). Competence-Based Student Modelling with Dynamic Bayesian Networks [Paper submitted for publication]. arXiv:2008.12114. https://arxiv.org/abs/2008.12114

Moreno Castañeda, M. (2015). La Educación Superior a Distancia en México. Una propuesta para su análisis histórico. En J. Zubieta García \& C. Rama Vitale (Eds.), La Educación a Distancia en México: Una nueva realidad universitaria (pp. 3-16). Universidad Nacional Autónoma de México. https://virtualeduca.org/documentos/observatorio/2015/la-educacion-a-distancia-enmexico.pdf

Moreno Castañeda, M., \& Pérez Alcalá, M. del S. (Eds.). (2010). Modelo educativo del Sistema de Universidad Virtual. Universidad de Guadalajara.

Nkambou, R., Lefebvre, B., \& Gauthier, G. (1996). A Curriculum-Based Student Model for Intelligent Tutoring Systems. Proceedings of the Fifth International Conference

Mapas de competencias genéricas del bachillerato. Rafael Morales, Morales Gamboa, González Navarro y Reyes Sánchez.

Página 25 de 27 
on User Modelling, 91-98.

Object Management Group. (2003). OMG Unified Modeling Language Specification, Version 1.5. Object Monagement Group. https://www.omg.org/spec/UML/1.5

Paquette, G. (2007). Ontology and Framework for Knowledge and Competency Management. Knowledge Creation Diffusion Utilization.

Paquette, G., Léonard, M., Lundgren-Cayrol, K., Mihaila, S., \& Gareau, D. (2006). Learning Design based on Graphical Knowledge-Modelling. Educational Technology \& Society, 9(1), 97-112.

Pépiot, G., Cheikhrouhou, N., Furbringer, J.-M., \& Glardon, R. (2007). UECML: Unified Enterprise Competence Modelling Language. Computers in Industry, 58, 130-142. https://doi.org/10.1016/j.compind.2006.09.010

Perrenoud, P. (2008). Construir competencias ¿es darle la espalda a los saberes? Revista de Docencia Universitaria, Número monográfico II: Formación centrada en competencias (II). http://revistas.um.es/redu/article/viewFile/35261/33781

Ramírez, C., \& Sánchez, E. (2012). A Model for the Representation of Competences Applied to Student's Knowledge Modelling. Research in Computing Science, 56, 7785.

Rodríguez Yépez, M., \& Morales Gamboa, R. (2019). Modelado de niveles de competencia por un sistema inteligente. Memorias CIIE 2019, 1078-1082.

Różewski, P., \& Małachowski, B. (2009). Competence Management in KnowledgeBased Organisation: Case Study Based on Higher Education Organisation. En R. Goebel, J. Siekmann, \& W. Wahlster (Eds.), Knowledge Science, Engineering and Management (pp. 358-369). Springer. https://doi.org/10.1007/978-3-642-104886_35

Różewski, P., \& Małachowski, B. (2010). System for Creative Distance Learning Environment Development Based on Competence Management. En R. Setchi, I. Jordanov, R. J. Howlett, \& L. C. Jain (Eds.), Knowledge-Based and Intelligent Information and Engineering Systems: Vol. Part IV (pp. 180-189). Springer. https://doi.org/10.1007/978-3-642-15384-6_20

Sampson, D., \& Fytros, D. (2008). Competence Models in Technology-Enhanced Competence-Based Learning. En H. H. Adelsberger, Kinshuk, J. M. Pawlovski, \& D. Sampson (Eds.), Handbook on Information Technologies for Education and Training (2a ed., pp. 155-177). Springer. https://link.springer.com/book/10.1007\%2F978-3540-74155-8

Sampson, D. G. (2009). Competence-related Metadata for Educational Resources that Support Lifelong Competence Development Programmes. Educational Technology \& Society, 12(4), 149-159.

Sampson, D., Karampiperis, P., \& Fytros, D. (2007). Developing a common metadata model for competencies description. Interactive Learning Environments, 15(2), 137150. https://doi.org/10.1080/10494820701343645

Secretaría de Educación Pública. (2008, octubre 21). ACUERDO número 444 por el que se establecen las competencias que constituyen el marco curricular común del Sistema Nacional de Bachillerato. Diario Oficial de la Federación.

Mapas de competencias genéricas del bachillerato. Rafael Morales, Morales Gamboa, González Navarro y Reyes Sánchez. 
http://dof.gob.mx/nota_detalle.php?codigo $=5064951 \&$ fecha $=21 / 10 / 2008$

Sistema de Universidad Virtual. (2021). Bachillerato General por Áreas Interdisciplinarias (BGAI) [Página institucional]. Sistema de Universidad Virtual. http://udgvirtual.udg.mx/bachillerato

Sitthisak, O., Gilbert, L., Davis, H. C., \& Gobbi, M. (2007). Adapting health care competencies to a formal competency model. ICALT 2007 - Proceedings of the 7th IEEE International Conference on Advanced Learning Technologies, 172-174.

Stoof, A., Martens, R. L., \& Merriënboer, J. J. G. van. (2007). Web-based support for constructing competence maps: Design and formative evaluation. Educational Technology Research and Development, 55(4), 347-368. https://doi.org/10.1007/s11423-006-9014-5

VanLehn, K. (1988). Student Modelling. En M. C. Polson \& J. J. Richardson (Eds.), Foundations of Intelligent Tutoring Systems (pp. 55-78). Lawrence Erlbaum Associates.

Vasconcelos, J. Â. B., Kimble, C., Ribeiro Gouveia, F., \& Kudenko, D. (2001). Reasoning in corporate memory systems: A case study of group competencies. Proceedings of the 8th International Symposium on the Management of Industrial and Corporate Knowledge. 8th International Symposium on the Management of Industrial and Corporate Knowledge, Compiègne, France.

Wang, H.-F., \& Wang, C. H. (2018). Modelling of Optimal Expansion of a Fuzzy Competence Set. International Transactions in Operational Research, 5(5), 413-424. https://doi.org/10.1111/j.1475-3995.1998.tb00124.x

World Wide Web Consortium. (2016, octubre 11). Extensible Markup Language (XML). World Wide Web Consortium (W3C). https://www.w3.org/XML/

Yu, P. L., \& Zhang, D. (1990). A foundation for competence set analysis. Mathematical Social Sciences, 20(3), 251-299. https://doi.org/10.1016/0165-4896(90)90005-R

Zone of Proximal Development. (2009). En D. Matsumoto (Ed.), The Cambridge Dictionary of Psychology. Cambridge University Press.

Mapas de competencias genéricas del bachillerato. Rafael Morales, Morales Gamboa, González Navarro y Reyes Sánchez. 